\title{
Diversity of anopheline species and their Plasmodium infection status in rural Bandarban, Bangladesh
}

Mohammad Shafiul Alam, Sumit Chakma', Wasif A Khan ${ }^{1}$, Gregory E Glass, Abu Naser Mohon ${ }^{2}$, Rubayet Elahi', Laura C Norris' ${ }^{2}$, Milka Patracia Podder', Sabeena Ahmed', Rashidul Haque', David A Sack², David J Sullivan Jr ${ }^{2}$ and Douglas E Norris ${ }^{2}$

\begin{abstract}
Background: Historically, the Chittagong Hill Tracts (CHT) of Bangladesh was considered hyperendemic for malaria. To better understand the contemporary malaria epidemiology and to develop new and innovative control strategies, comprehensive epidemiologic studies are ongoing in two endemic unions of Bandarban district of CHT. Within these studies entomological surveillance has been undertaken to study the role of the existing anopheline species involved in the malaria transmission cycle throughout the year.

Methods: CDC miniature light traps were deployed to collect anopheline mosquitoes from the sleeping room of the selected houses each month in a single union (Kuhalong). Molecular identification was carried out for available Anopheles species complexes. Circumsporozoite proteins (CSP) for Plasmodium falciparum, Plasmodium vivax-210 (Pv-210) and Plasmodium vivax-247(Pv-247) were detected by Enzyme-linked immunosorbent assay (ELISA) from the female anopheline mosquitoes. To confirm CSP-ELISA results, polymerase chain reaction (PCR) was also performed.

Results: A total of 2,837 anopheline mosquitoes, of which 2,576 were female, belonging to 20 species were collected from July 2009-June 2010. Anopheles jeyporiensis was the most abundant species (18.9\%), followed by An. vagus (16.8\%) and An. kochi (14.4\%). ELISA was performed on 2,467 female mosquitoes of 19 species. 15 (0.6\%) female anophelines belonging to eight species were found to be positive for Plasmodium infection by CSP-ELISA. Of those, $11(0.4 \%)$ mosquitoes were positive for P. falciparum and four (0.2\%) for Pv-210. No mosquito was found positive for Pv-247. An. maculatus $(2.1 \%, 2 / 97)$ had the highest infection rate followed by An. umbrosus (1.7\%, 2/115) and An. barbirostris (1.1\%,2/186). Other infected species were An. nigerrimus, An. nivipes, An. jeyporiensis, An. kochi, and An. vagus. Out of 11 P. falciparum CSP positive samples, seven turned out to be positive by PCR. None of the samples positive for Pv-210 was positive by PCR. In terms of abundance and incrimination, the results suggest that An. maculatus, An. jeyporiensis and An. nivipes play important roles in malaria transmission in Kuhalong.

Conclusion: The findings of this study suggest that even in the presence of an insecticide impregnated bed-net intervention, a number of Anopheles species still play a role in the transmission of malaria. Further investigations are required to reveal the detailed biology and insecticide resistance patterns of the vector mosquito species in endemic areas in Bangladesh in order to assist with the planning and implementation of improved malaria control strategies.
\end{abstract}

\footnotetext{
* Correspondence: shafiul@icddrb.org

${ }^{1}$ International Centre for Diarrhoeal Disease Research Bangladesh (icddr,b),

Dhaka, Bangladesh

Full list of author information is available at the end of the article
} 


\section{Background}

In Bangladesh, malaria is most endemic in the highland areas bordering India and Myanmar. Of the 13 malaria endemic districts in Bangladesh, the Chittagong Hill Tracts (CHT) are composed of three districts; Rangamati, Bandarban and Khagrachhari, which contribute $80 \%$ of the country's total malaria case burden [1]. Plasmodium falciparum and $P$. vivax are the two main parasites in CHT [1,2] although some sporadic reports of $P$. malariae and $P$. ovale have been reported recently $[3,4]$. Malaria incidence is seasonal in Bangladesh where the warm and wet months of May-October define the peak season and the dry and cooler months of NovemberApril define the off season [5].

To date, 35 anopheline species have been reported in Bangladesh [6]. Of those, four species, An. baimaii (dirus D), An. minimus, An. sundaicus and An. philippinensis were incriminated as malaria vectors during the Malaria Eradication Programme (MEP) of the 1960's. In the early 1990's three additional species (An. aconitus, An. annularis and An. vagus) were incriminated following three respective outbreaks in flood plain areas of the country $[7,8]$. However, due to loss of forest habitat, the density of two 'primary vectors' (An. baimaii and An. minimus) were dramatically reduced in several sporadic entomological investigations carried out by the Malaria and Parasitic Disease Control Unit (M\&PDC) (personal communication with N. Chaudhury, Senior Entomologist, M\&PDC).

An entomological investigation carried out in three malaria endemic border areas of the country during the peak of the 2009 transmission season found seven Anopheles species harboring Plasmodium based on ELISA. Except for An. philippinensis and An. vagus five other species (An. karwari, An. maculatus, An. barbirostris, An. nigerrimus, and An. subpictus), not previously incriminated in Bangladesh, were found to be infected [8]. From that study it was concluded that in the absence of recognized 'primary malaria vectors', other Anopheles species might play a significant role in continued malaria transmission in Bangladesh.

In collaboration with the Johns Hopkins Malaria Research Institute (JHMRI), icddr,b initiated a study to understand the epidemiology of malaria transmission in two unions (Kuhalong and Rajbila) of Bandarban district in mid 2009. The method has been described elsewhere [9]. The distribution of the anopheline fauna and the species' roles in the malaria transmission cycle in the study areas during both the wet and dry season remain the main focus of the current entomological investigations. The aim of this paper is to report the faunal diversity and Plasmodium infection status of anopheline species that have been collected in the first year (July 2009-June 2010) of the entomological surveillance in Kuhalong.

\section{Methods}

\section{Study area}

Kuhalong (22 $\left.12^{\prime} 45^{\prime \prime} \mathrm{N}, 92^{\circ} 9^{\prime} 35^{\prime \prime} \mathrm{E}\right)$ is located adjacent to the Bandarban town. Kuhalong was divided into 12 clusters, which had roughly equal numbers of households as part of the epidemiologic study. A description of Kuhalong and its clusters have been provided earlier [9]. In brief, the union has a total area of $79 \mathrm{sq} \mathrm{km}$ with a population of more than 11,390 . Kuhalong is a hilly and forested area with an average elevation of 80 meters ranging up to 152 meters. Some other important features of Kuhalong include the presence of intricate branches of rivers, several streams, marshy lands and plant monoculture (teak and rubber). The land for plant monoculture was created by destroying natural forest. 99.5\% of the inhabitants of the area have bed net coverage (untreated/insecticide treated). But when asked, 10\% of the respondents admitted that they had not used a bed net the previous night (unpublished data).

\section{Collection of Anopheles mosquitoes}

CDC miniature light trap (model 512, John W. Hock Inc, USA) was deployed for indoor mosquito collection from the sleeping room. Traps were placed for 12 hours (6 pm to $6 \mathrm{am}$ ). During the wet season 100 houses were selected randomly, based on elevation and were trapped. This was reduced to 50 houses in the off (dry) season. Later, when trapping started in the second Union (Rajbila), the number was fixed to 60 houses at each union (5 houses in each of 12 clusters) due to the available resources and personnel. Each house was later trapped once in a month throughout the season in order to obtain longitudinal information.

\section{Mosquito sample preparation}

Mosquitoes were collected from the light traps using a battery-powered aspirator and were subsequently killed by chloroform. Anopheline mosquitoes were sorted and preserved temporarily in plastic tubes. After identifying the species at the field office in Bandarban each mosquito was preserved in a separate plastic vial $(1.5 \mathrm{ml})$ labelled and capped with silica gel and cotton.

\section{Circumsporozoite protein (CSP) ELISA}

The ELISA methods were described previously elsewhere $[10,11]$. ELISA distinguished between circumsporozoite protein (CSP) of P. falciparum and two distinct polymorphs of P. vivax: $\mathrm{Pv}-210$ and $\mathrm{Pv}-247$. In each test, field caught male Anopheles spp. was used as the negative control. The positive controls and monoclonal antibody (MAB) for the ELISAs were obtained from the Centers for Disease Control and Prevention, Atlanta, USA. The optical density (OD) was measured at $410 \mathrm{~nm}$ in a Bio-Rad ELISA plate reader, 60 minutes after adding 
the substrate [8]. A cut-off value at least twice the mean OD of the negative controls was considered as positive. ELISA positive samples in a given day were repeated the next day to confirm the result.

\section{PCR}

Remaining mosquito lysates were used for DNA extraction using the QiaAmp DNA Minikit (Qiagen, Valencia, CA), following the tissue extraction protocol. Nested PCR was performed to detect $P$. falciparum and $P$. vivax infection from extracted mosquito DNA using the primers described by Snounou et al. [12]. PCR conditions were modified to adjust for low concentration of DNA from mosquito samples. $2 \mu \mathrm{L}$ of the amplicon from the first step was used as template in the second step.

To confirm taxonomic identification of some Anopheles species, a confirmatory PCR was made available, based on availability of the established protocols. Protocols used were Phuc et al. [13] for species belonging to An. minimus and Myzomia Series, two different protocols of Walton et al. [14,15] for Annularis and Maculatus groups, Huong et al. [16] for An. dirus sibling species, and Goswami et al. [17] for the An. culicifacies complex, respectively. Protocols were followed with some modifications. DNA amplifications were done on S1000 ${ }^{\circledR}$ Thermal Cycler (Bio-Rad Laboratories, Inc., Hercules, CA) and visualized under UV illumination after electrophoresis on ethidium bromide-stained 1.5\% agarose gels along with invitrogen ${ }^{\circledR} 100$ base pair (bp) molecular mass marker (Life technologies, NY, USA). Representative samples (DNA from homogenates) were sent for sequencing to the JHMRI. The list of primers is given in Additional file 1.

\section{Sequencing of mosquito specimens}

The variable internal transcribed spacer 2 region (ITS2) was amplified with primers from the flanking $5.8 \mathrm{~S}$ and 28 S genes; ITS2A (5'-TGT GAA CTG CAG GAC ACA T-3') and ITS2B (5'-ACC CCC TGA ATT TAA GCA TA-3') [18]. Because sequencing reactions with

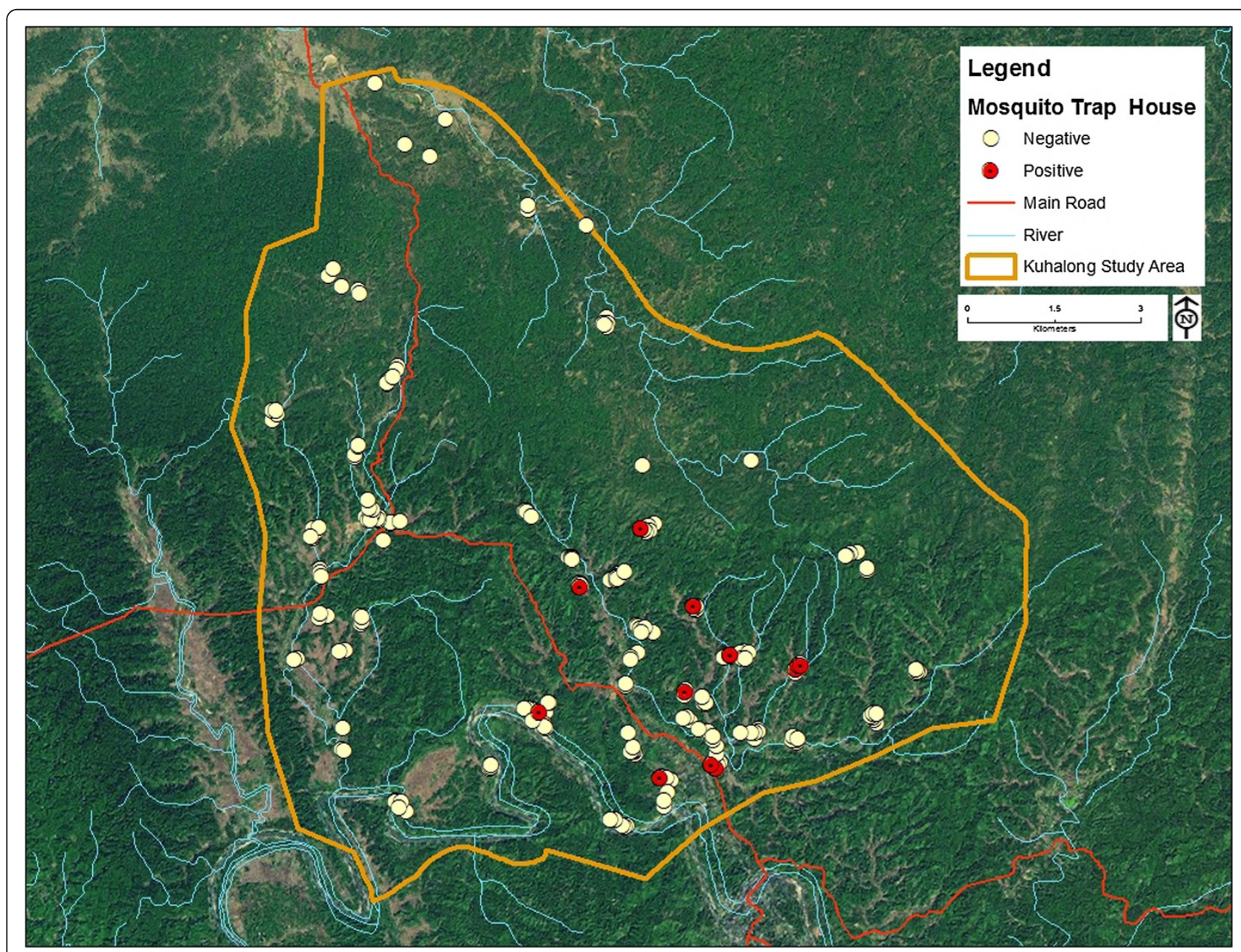

Figure 1 A satellite image over study area showing location of 268 trapping houses. Here, positive means houses that had CSP-positive mosquitoes and negative means houses with CSP-negative mosquitoes or without female anopheline mosquitoes. 
ITS2A frequently failed, some samples were amplified and sequenced using the novel primer ITS2B1 ( $5^{\prime}$-GTC CCT ACG TGC TGA GCT TC-3'). This primer binds further downstream in the $28 \mathrm{~S}$ gene; such that the $3^{\prime}$ portions of products immediately upstream of the ITS2B binding site, could be sequenced. Each $25 \mu \mathrm{L}$ reaction contained 1X PCR buffer, $200 \mu \mathrm{M}$ each dNTP, 30 pmol each primer, 2 units Taq polymerase, and $1.0 \mu \mathrm{L}$ DNA template. Products were amplified in a thermocycler (MJ Research, Watertown, MA, USA) using the following conditions: 2 minute initial denaturation at $94^{\circ} \mathrm{C}$, 40 cycles of 30 seconds at $94^{\circ} \mathrm{C}, 30$ seconds at $50^{\circ} \mathrm{C}$, and 40 seconds at $72^{\circ} \mathrm{C}$, and a 10 minute final extension at $72^{\circ} \mathrm{C}$. PCR product size ranged from 480 bp to 847 bp. $5 \mu \mathrm{L}$ of each PCR product was subjected to electrophoresis on a $2 \%$ agarose gel, stained with ethidium bromide, and visualized under UV illumination. The remainder of each successful PCR reaction was purified using a Qiaquick PCR prep kit (Qiagen, Valencia, CA). Products were sequenced in both directions using dye terminator chemistry on a 3730xl DNA Analyzer (Applied Biosystems, Foster City, CA) at the Johns Hopkins University School of Medicine Sequencing and Synthesis Facility.

\section{Results}

Collections were made during 641 trap nights from July 2009 to June 2010 in households throughout Kuhalong (Figure 1). A total of 2,837 Anopheles mosquitoes (2,576 female and 261 male) were caught (4.4 mosquitoes/trap night; std. error 0.21). Among the collected female anophelines, 20 species were confirmed based on taxonomic characteristics, adjusted by PCR for available species complexes. A representative half of the specimens belonging to An. philippinensis based on morphological characteristics were later confirmed as An. nivipes by PCR followed by sequencing. Similarly the identity of all An. pallidus samples (approximately 30 specimens) was revised to $A n$. nivipes after molecular confirmation. Therefore, all individual specimens originally morphologically identified as An. philippinensis and An. pallidus were listed as $A n$. nivipes in our final data set. A number of An. minimus were molecularly identified as $A n$. varuna, whereas the remaining $A n$. minimus specimens were confirmed as $A n$. minimus subspecies A. Three subspecies of An. culicifacies (B, $\mathrm{C}$ and $\mathrm{E}$ ) were confirmed, with dominance of subspecies B. A strong concordance was observed between morphological and moleculer detection for An. baimaii and An. maculatus group.

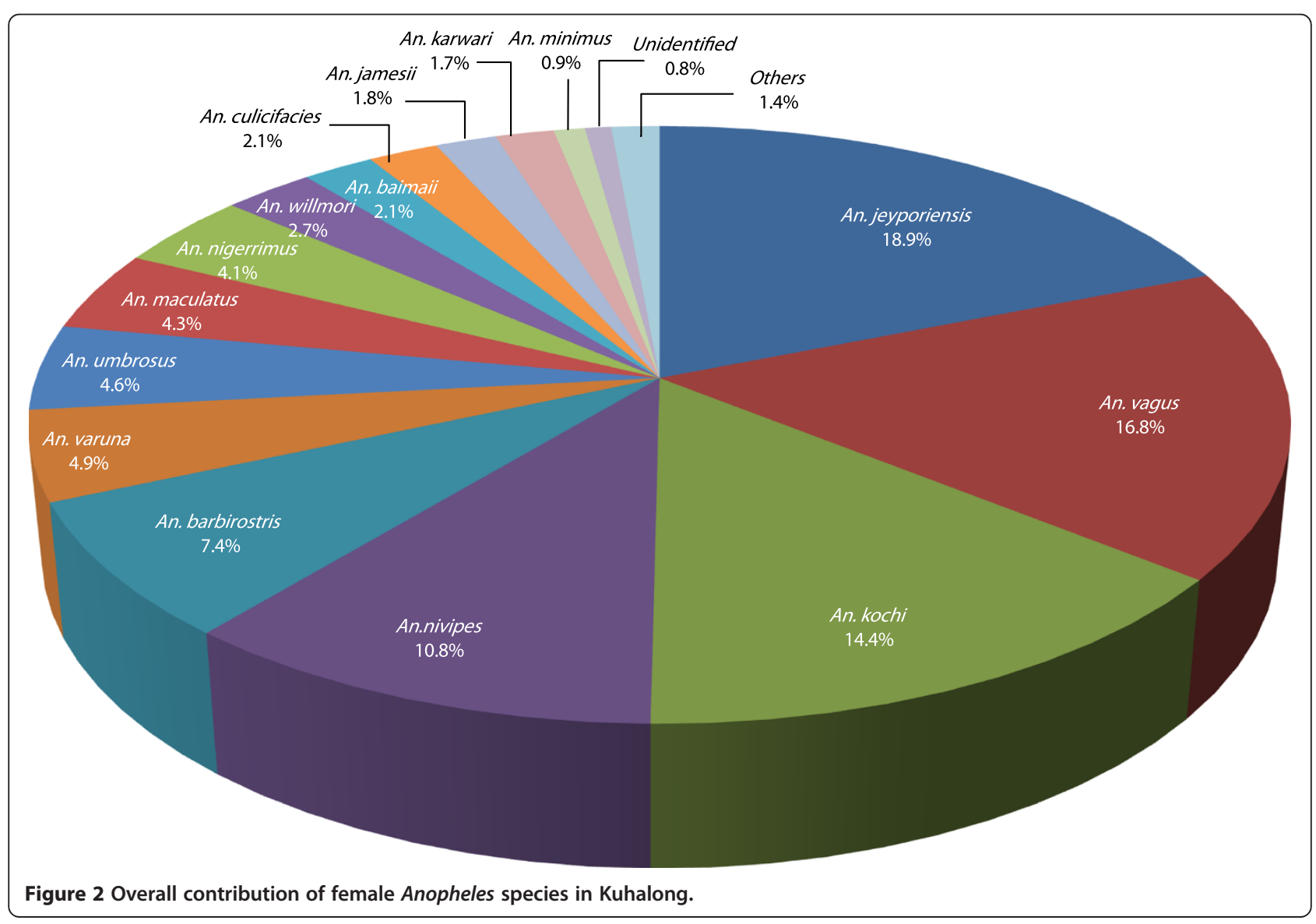


Three species were nearly co-dominant. An. jeyporiensis (18.9\%) was the most commonly captured species, followed by An. vagus (16.8\%) and An. kochi (14.4\%) (Figure 2). The relative abundance and dominance of anopheline species varied temporally throughout the year (Figure 3). Slightly more female anophelines were caught in the non-malaria dry season $(n=1391$, mean 4.3 mosquitoes/trap night) than peak wet transmission season ( $n=1185$, mean 3.7 mosquitoes/trap night). However, this seasonal difference in mosquito collection rates was not statistically significant $(\mathrm{p}>0.05)$. The highest diversity of species was found in July 2009 (16) and lowest in September 2009 and April 2010 (11). An. nivipes appeared to be the dominant species during peak malaria season. However, in the off season, $A n$. kochi was the dominant species. In both seasons, An. jeyporiensis and An. vagus were the second and third most numerous species, respectively. The density of An. nivipes complex was very low in the off season. An. maculatus, $A n$. nigerrimus, $A n$. umbrosus and $A n$. barbirostris appeared to retain a moderate density throughout the year (Figure 4).

CSP-ELISA was performed on 2,467 female anopheline mosquitoes identified to 19 species. The untested mosquitoes $(\mathrm{n}=109)$, including a single specimen of An. turkhudi, were kept as voucher specimens. Fifteen mosquitoes collected from 13 houses and belonging to eight species were Plasmodium-positive by CSP-ELISA. Thus, the overall infection rate was $0.6 \%(15 / 2467)$. By species, the highest infection rate was observed in $A n$. maculatus (2.1\%) followed by An. umbrosus (1.7\%), An. barbirostris (1.1\%), An. nigerrimus (1\%), An. nivipes (0.8\%), An. jeyporiensis (0.6\%), An. kochi (0.5\%), and An. vagus $(0.2 \%)$, respectively (Table 1$)$.
Among the CSP-positive anophelines, 11 (0.4\%) mosquitoes belonging to six species were found positive for P. falciparum and four (0.2\%) mosquitoes belonging to three species were found positive for Pv-210 (Table 2). None of the mosquitoes were positive for $\mathrm{Pv}-247$ or had mixed Plasmodium infection. P. falciparum positive Anopheles species included An. barbirostris, An. jeyporiensis, An. kochi, An. maculatus, An. nigerrimus, and An. nivipes. Pv-210 positive species included $A n$. nivipes, $A n$. umbrosus and An. vagus.

The highest monthly infection rates for Plasmodium were observed in October 2009 (2\%) and November 2009 (1.3\%). Plasmodium-infected mosquitoes were not detected in September or December of 2009 and AprilJune 2010 (Figure 5). In the dry season three sporozoite positive mosquitoes were caught in November 2009 and two in March 2010. Although the infection rate in peak season $(0.8 \%)$ was twice that of off season $(0.4 \%)$, this seasonal difference in mosquito infection rates was not statistically significant ( $\mathrm{p}>0.05)$.

Of the 15 Plasmodium-positive mosquitoes, seven of these were positive for the presence of P. falciparum by nested PCR out of 11 that tested positive by CSP-ELISA. However, none of the Pv-210 CSP positive samples were positive by PCR. Species that had both CSP and PCR positive samples included five species: An. maculatus, An. jeyporiensis, An. nivipes, An. barbirostris and An. nigerrimus (Table 2).

\section{Discussion}

The Anopheles species diversity is very high in Kuhalong. This diversity is even higher than what had been observed in a previous study in Matiranga, a sub-district of $\mathrm{CHT}$, where 15 anopheline species were reported in a

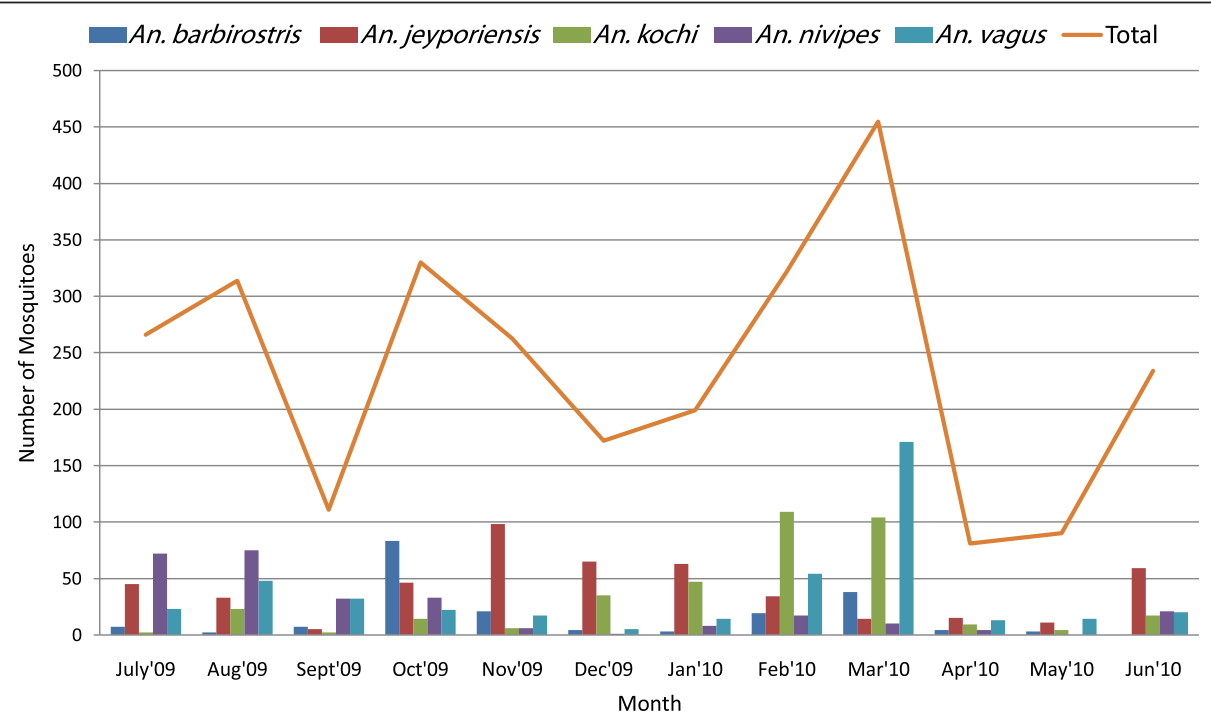

Figure 3 Monthly frequency of five prevalent female Anopheles species in Kuhalong. 


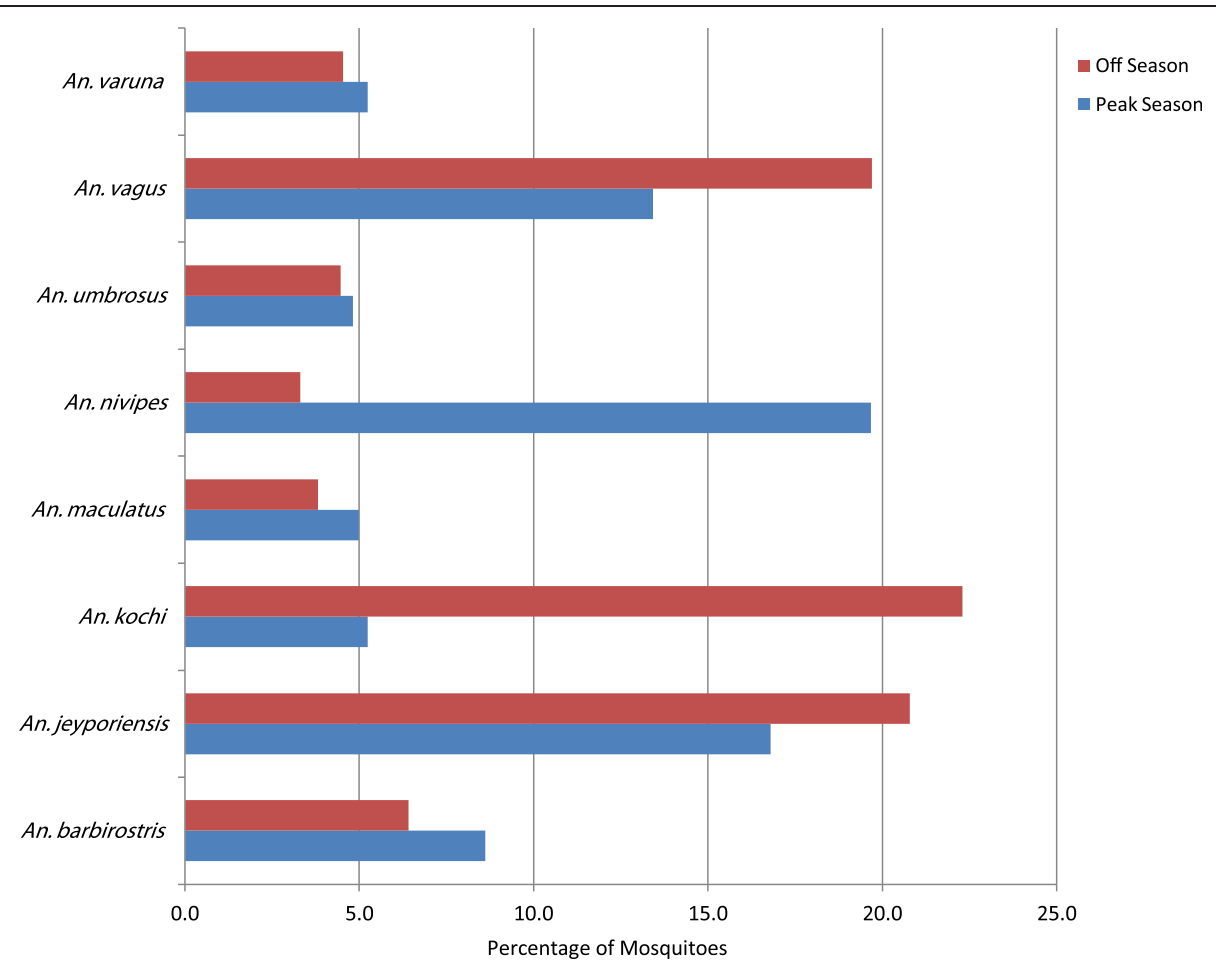

Figure 4 Contribution of eight prevalent female Anopheles species according to transmission season.

peak transmission season, of which seven were positive for CSP [8]. The greater number of anopheline species collected in Kuhalong might be due to the cumulative year-long trapping effort, as several species were most frequently sampled during the low transmission season (Figures 3 and 4). This highlights the importance of off season trapping.

Of the eight Plasmodium-infected anopheline species reported in this study, An. jeyporiensis and An. kochi have never been previously incriminated as vectors in Bangladesh. However, An. jeyporiensis and An. kochi have been considered to be potential vectors in the foothills of Assam, a north-eastern Indian state [19]. There is also a report of sporozoites in An. jeyporiensis made in 1944 by Macan from mosquitoes collected on the Myanmar-Bangladesh border [20]. The high relative abundance of An. jeyporiensis and its infection confirmed by CSP followed by PCR indicates that this species likely plays an important role in the malaria transmission in the area.

Although An. maculatus is considered an important vector in Peninsular Malaysia and Southern Thailand $[21,22]$, it was thought to be a less important species in Bangladesh earlier. This species was first found to be CSP infected in Bangladesh in recent times on the basis of a single positive mosquito out of seven tested [8]. Our results re-emphasize its potential importance as a vector in this region.
Despite expectations based on prior observations, no An. baimaii or An. minimus s.l. were found CSP-positive during this study in Kuhalong. Further study is required, but these observations may indicate that these species are less abundant, are locally less competent or that this reflects a foraging effect/trapping bias. Similarly, no $A n$. culicifacies, a major malaria vector in some parts of India and Sri Lanka $[23,24]$, were found to be infected with Plasmodium in Kuhalong. It is worth noting that An. culicifacies is primarily represented by species B in Kuhalong, a species considered to be a non-vector or a poor vector $[24,25]$, and it is common in the eastern states of India sharing borders with Bangladesh [26]. This supports the idea that this species does not have a vectorial role in Kuhalong. Apart from An. vagus, two other potential secondary malaria vectors in Bangladesh, An. aconitus and An. annularis $[27,28]$, were rarely collected in Kuhalong and all were negative for Plasmodium infection by CSP ELISA.

An. philippinensis has historically been considered the main vector of malaria in the vast plain areas of Bangladesh, but has been considered rare in the CHT [7]. The findings of the present study, in accordance with other recent observations [8], initially suggested that this species has adapted to the high land areas and has expanded its role in the transmission of malaria. However, An. nivipes is morphologically very similar with $A n$. philippinensis and often confused with the latter. The 
Table 1 Prevalence of female anopheline mosquitoes and their CSP positive rates

\begin{tabular}{llllll}
\hline SI & Species & No. collected & No. tested & Positive & Pre (\%) \\
\hline 1 & An. aconitus & 19 & 18 & 0 & 0 \\
\hline 2 & An. annularis & 2 & 2 & 0 & 0 \\
\hline 3 & An. baimaii & 55 & 44 & 0 & 0 \\
\hline 5 & An. barbirostris & 191 & 186 & 2 & 1.1 \\
\hline 6 & An. culicifacis & 54 & 54 & 0 & 0 \\
\hline 7 & An. jamesii & 47 & 45 & 0 & 0 \\
\hline 8 & An. karwari & 45 & 479 & 3 & 0.6 \\
\hline 9 & An. kochi & 372 & 42 & 0 & 0 \\
\hline 10 & An. maculatus & 112 & 369 & 2 & 0.5 \\
\hline 11 & An. minimus & 24 & 97 & 2 & 2.1 \\
\hline 12 & An. nigerrimus & 105 & 18 & 0 & 0 \\
\hline 13 & An. nivipes & 279 & 264 & 2 & 1 \\
\hline 14 & An. subpictus & 8 & 6 & 0 & 0 \\
\hline 15 & An. tessellatus & 7 & 6 & 0 & 0 \\
\hline 16 & An. turkhudi & 1 & 0 & 0 & 0 \\
\hline 17 & An. umbrosus & 119 & 115 & 2 & 1.7 \\
\hline 18 & An. vagus & 433 & 429 & 1 & 0.2 \\
\hline 19 & An. varuna & 125 & 121 & 0 & 0 \\
\hline 20 & An. willmori & 70 & 68 & 0 & 0 \\
\hline 21 & Unidentified & 20 & 0 & 0 & 0 \\
\hline & Total & 2576 & 2467 & 15 & 0.6 \\
\hline & & & & \\
\hline
\end{tabular}

adults of these two species are differentiated by a single characteristic related to a pre-sector dark mark on the wing vein [29]. In the Assam State of India, recent studies document the role of the An. philippinensis-nivipes complex in malaria transmission $[19,30]$ and later confirmed the presence of $P$. falciparum DNA in two $A n$. nivipes specimens, that were molecularly identified [31]. The presence of $P$. falciparum specific CSP by ELISA and later parasite DNA in An. nivipes in the present study support the previous observations in India. These two species have never been differentiated in Bangladesh until this report.

Representatives of $A n$. barbirostris and An. nigerrimus species were found to be positive for the presence of CSP, which was also seen in previous observations made in the CHT [8]. Previously in this region, An. barbirostris was confirmed as a vector in Sri Lanka based on CSP-ELISA [32].

Depletion of the forest habitats, preferred by $A n$. baimaii and $A n$. minimus and conversion to agricultural use may have allowed members of the An. nivipes to extend their range into highland areas. It is unknown how these species interact, whether it is the habitat that drives the relative abundance of these species or whether there is active competition and replacement as well.
Thus, the bionomics of anopheline species requires further study and will reveal valuable information necessary for future control strategies.

As a method, CSP-ELISA has several advantages although it is thought to be less sensitive, particularly when low numbers of sporozoites are present [33]. Hence, a false positive result is also possible due to the presence of CSP in body parts other than salivary glands $[33,34]$ and also due to the presence of bovine or swine blood [35]. On the other hand, PCR is more sensitive than ELISA and can detect fewer parasites [36], but is not sporozoite specific and is recommended to reconfirm CSP-ELISA results [37]. None of the Pv-210 CSP positive samples in the study tested positive by PCR. A low concentration of DNA obtained from CSP homogenates could be another reason for negative PCR results along with CSP false positive samples.

In terms of incrimination by overall abundance and infection rates, $A n$. maculatus, An. jeyporiensis and $A n$. nivipes all are likely to have important roles in malaria transmission in Kuhalong. This is not to say that other species do not also contribute significantly to malaria transmission in Kuhalong.

The National Malaria Control Programme (NMCP) of Bangladesh initiated a programme in 2007 with financial support from the Global Fund. This programme includes diagnosis and treatment, distribution of long-lasting insecticidal bed net (LLIN) and re-treatment of insecticide-treated nets (ITNs). As of 2010, the NMCP has treated/retreated more than 2 million bed nets with

Table 2 Summary information of CSP positive Anopheles female mosquitoes from Kuhalong, Bandarban

\begin{tabular}{lllll}
\hline ID & Species Name & CSP ELISA $^{\mathbf{a}}$ & PCR $^{\mathbf{b}}$ & Fed $^{\mathbf{c}}$ \\
\hline An-638 & An. nigerrimus & Pf & Pf & 0 \\
\hline An-677 & An. maculatus & Pf & Pf & 0 \\
\hline $09-0064$ & An. maculatus & Pf & Pf & 0 \\
\hline $09-0376$ & An. jeyporiensis & Pf & Pf & 1 \\
\hline $09-0380$ & An. nivipes & Pf & Pf & 0 \\
\hline $09-0381$ & An. kochi & Pf & 0 & 1 \\
\hline $09-0396$ & An. vagus & Pv-210 & 0 & 0 \\
\hline $09-0439$ & An. barbirostris & Pf & Pf & 0 \\
\hline $09-0459$ & An. umbrosus & Pv-210 & 0 & 1 \\
\hline $09-0775$ & An. jeyporiensis & Pf & 0 & 0 \\
\hline $09-0785$ & An. umbrosus & Pv-210 & 0 & 1 \\
\hline $09-0826$ & An. barbirostris & Pf & 0 & 0 \\
\hline $10-1147$ & An. jeyporiensis & Pf & Pf & 0 \\
\hline $10-1700$ & An. kochi & Pf & 0 & 0 \\
\hline $10-1917$ & An. nivipes & Pv-210 & 0 & 1 \\
\hline Pf & & & 0 \\
\hline
\end{tabular}

${ }^{\mathrm{a}} P f=P$. falciparum, $\mathrm{Pv}-210=P$. vivax -210.

${ }^{\mathrm{b}} P f=P$. falciparum (+ve), $0=$ negative.

${ }^{\mathrm{C}} \mathrm{O}=$ unfed, $1=$ blood fed. 


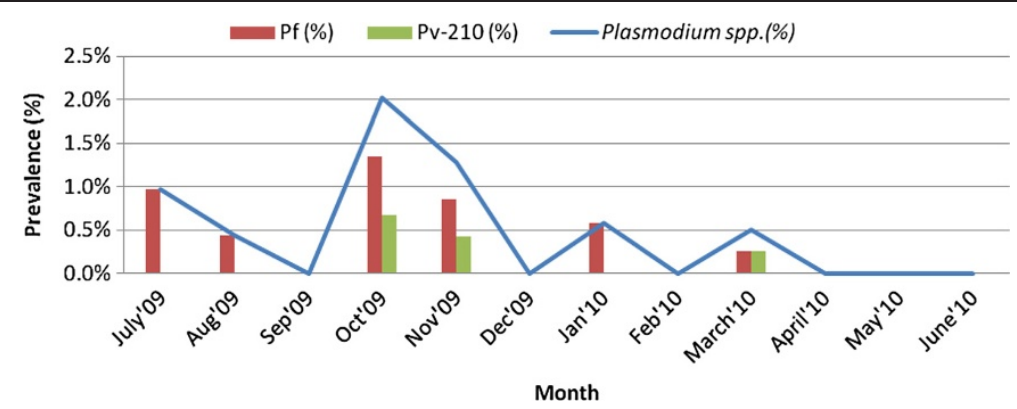

Figure 5 Monthly variation in CSP positive rates among anopheline species in Kuhalong.

insecticides and distributed almost 1.7 million LLINs throughout the malaria endemic areas of the country [38]. However, no prominent work has been reported so far to monitor the resistance patterns of the anopheline mosquitoes against the insecticides used in these control efforts. The high diversity of anophelines, may increase the risk of insecticide resistance and could alter biting behaviours which may circumvent some of the protection from bednets. This high species diversity is typical of Southeast Asia, in contrast to many parts of Africa where only a few species are major contributors to Plasmodium transmission.

\section{Conclusions}

The findings of this study illustrate that even in areas of Bangladesh where ITNs and LLINs have been deployed as malaria interventions, a number of diverse Anopheles species still play a role in the transmission of malaria. Further study of the bionomics, ecology, and insecticide resistance of these species is necessary to understand the transmission biology and to provide the information required for the development of evidence-based control programmes in Bangladesh. This insight may be useful to other countries similarly facing high burdens of malaria.

\section{Additional file}

\section{Additional file 1: List of primers.}

\section{Competing interests}

The authors declare that they have no competing interests.

\section{Authors' contributions}

MSA, WK, SC, GEG, DAS, SA, RH, DJS, DEN conceived and designed the study. MSA and DEN supervised vector surveillance with assistance of SC. SC, RE, and MSA identified mosquito species. ANM, RE and MPP performed laboratory assays. LCN performed sequencing. MSA, DEN, GEG, ANM, RE, SA, DAS, and DJS analyzed the data. MSA, DJS and DEN drafted the manuscript. All authors read and approved the final version of the manuscript.

\section{Acknowledgements}

This research is funded by the Johns Hopkins Malaria Research Institute of Johns Hopkins Bloomberg School of Public Health (Grant No. 00679). icddr,b acknowledges with gratitude the commitment of Johns Hopkins University,
Baltimore, MD, USA, to its research efforts. We are grateful to the staff of the Bandarban field office for their active participation during entomological investigations. We are also grateful to Jacob Khyang, Chai Shwai Prue, Khaja Mohiuddin, Timothy Shields, Md. Sharif Hossain, and Humayun Kabir for their contributions to this study.

\section{Author details}

${ }^{1}$ International Centre for Diarrhoeal Disease Research Bangladesh (icddr,b), Dhaka, Bangladesh. ${ }^{2} J o h n s$ Hopkins Malaria Research Institute, Johns Hopkins Bloomberg School of Public Health, Baltimore, MD 21205, USA.

Received: 20 June 2012 Accepted: 15 July 2012

Published: 27 July 2012

\section{References}

1. Haque U, Ahmed SM, Hossain S, Huda M, Hossain A, Alam MS, Mondal D, Khan WA, Khalequzzaman M, Haque R: Malaria prevalence in endemic districts of Bangladesh. PLOS One 2009, 4(8):e6737.

2. Alam MS, Mohon AN, Mustafa S, Khan WA, Islam N, Karim MJ, Khanum H, Sullivan DJ Jr, Haque R: Real-time PCR assay and rapid diagnostic tests for the diagnosis of clinically suspected malaria patients in Bangladesh. Malaria J 2011, 10(1):175.

3. Fuehrer HP, Starzengruber P, Swoboda P, Khan WA, Matt J, Ley B, Thriemer K, Haque R, Yunus EB, Hossain SM, et al: Indigenous Plasmodium ovale malaria in Bangladesh. Am J Trop Med Hyg 2010, 83(1):75-78.

4. Rahman W, Chotivanich K, Silamut K, Tanomsing N, Hossain A, Faiz MA, Dondorp AM, Maude RJ: Plasmodium malariae in Bangladesh. Trans R SoC Trop Med Hyg 2010, 104(1):78-80.

5. Rahman A, Kogan F, Roytman L: Analysis of malaria cases in Bangladesh with remote sensing data. Am J Trop Med Hyg 2006, 74(1):17-19.

6. Jannat KN, Ahmed TU: The mosquito fauna, including new records of St. Martin's island, Bangladesh. Bangladesh. J Entomo/ 2006, 16(1):67-81.

7. Elias M, Dewan ZAR, Ahmed R: Vectors of malaria in Bangladesh. J Prev Social Med 1982, 1:20-28.

8. Alam MS, Khan MG, Chaudhury N, Deloer S, Nazib F, Bangali AM, Haque R: Prevalence of anopheline species and their Plasmodium infection status in epidemic-prone border areas of Bangladesh. Malaria J 2010, 9:15.

9. Khan WA, Sack DA, Ahmed S, Prue CS, Alam MS, Haque R, Khyang J, Ram M, Nyunt MM, Norris D, et al: Mapping hypoendemic, seasonal malaria in rural Bandarban, Bangladesh: a prospective surveillance. Malaria J 2011, 10:124.

10. Wirtz RA, Burkot TR, Graves PM, Andre RG: Field evaluation of enzymelinked immunosorbent assays for Plasmodium falciparum and Plasmodium vivax sporozoites in mosquitoes (Diptera: Culicidae) from Papua New Guinea. J Med Entomol 1987, 24(4):433-437.

11. Wirtz RA, Zavala F, Charoenvit Y, Campbell GH, Burkot TR, Schneider I, Esser KM, Beaudoin RL, Andre RG: Comparative testing of monoclonal antibodies against Plasmodium falciparum sporozoites for ELISA development. Bull World Health Organ 1987, 65(1):39-45.

12. Snounou G, Viriyakosol S, Zhu XP, Jarra W, Pinheiro L, do Rosario VE, Thaithong S, Brown KN: High sensitivity of detection of human malaria parasites by the use of nested polymerase chain reaction. Mol Biochem Parasitol 1993, 61(2):315-320. 
13. Phuc HK, Ball AJ, Son L, Hanh NV, Tu ND, Lien NG, Verardi A, Townson H: Multiplex PCR assay for malaria vector Anopheles minimus and four related species in the Myzomyia Series from Southeast Asia. Med Vet Entomol 2003, 17(4):423-428.

14. Walton C, Somboon P, Harbach RE, Zhang S, Weerasinghe I, O'Loughlin SM, Phompida S, Sochantha T, Tun-Lin W, Chen B, et al: Molecular identification of mosquito species in the Anopheles annularis group in southern Asia. Med Vet Entomol 2007, 21(1):30-35.

15. Walton $C$, Somboon $P$, O'Loughlin SM, Zhang S, Harbach RE, Linton YM, Chen B, Nolan K, Duong S, Fong MY, et al: Genetic diversity and molecular identification of mosquito species in the Anopheles maculatus group using the ITS2 region of rDNA. Infect Genet Evol 2007, 7(1):93-102.

16. Huong NT, Sonthayanon P, Ketterman AJ, Panyim S: A rapid polymerase chain reaction based method for identification of the Anopheles dirus sibling species. Southeast Asian J Trop Med Public Health 2001, 32(3):615-620

17. Goswami G, Singh OP, Nanda N, Raghavendra K, Gakhar SK, Subbarao SK: Identification of all members of the anopheles culicifacies complex using allele-specific polymerase chain reaction assays. Am J Trop Med Hyg 2006, 75(3):454-460.

18. Koekemoer LL, Kamau L, Hunt RH, Coetzee M: A cocktail polymerase chain reaction assay to identify members of the Anopheles funestus (Diptera: Culicidae) group. Am J Trop Med Hyg 2002, 66(6):804-811.

19. Prakash A, Bhattacharyya DR, Mohapatra PK, Mahanta J: Role of the prevalent Anopheles species in the transmission of Plasmodium falciparum and P. vivax in Assam state, north-eastern India. Ann Trop Med Parasitol 2004, 98(6):559-568.

20. Macan TT: Malaria survey of the Arakan region of Bengal and Burma. Parasitology 1950, 40(3-4):290-297.

21. Loong KP, Chiang GL, Eng KL, Chan ST, Yap HH: Survival and feeding behaviour of Malaysian strain of Anopheles maculatus Theobald (Diptera: Culicidae) and their role in malaria transmission. Tropical Biomedicine 1990, 7(1):71-76

22. Vythilingam I, Foo LC, Chiang GL, Chan ST, Eng KL, Mahadevan S, Mak JW, Singh Kl: The impact of permethrin impregnated bednets on the malaria vector Anopheles maculatus (Diptera: Culicidae) in aboriginal villages of Pos Betau Pahang, Malaysia. Southeast Asian J Trop Med Public Health 1995, 26(2):354-358

23. Ramasamy R, Ramasamy MS, Wijesundera DA, Wijesundera AP, Dewit I, Ranasinghe C, Srikrishnaraj KA, Wickremaratne C: High seasonal malaria transmission rates in the intermediate rainfall zone of Sri Lanka. Ann Trop Med Parasitol 1992, 86(6):591-600.

24. Subbarao SK: The Anopheles culicifacies complex and control of malaria. Parasitol Today 1988, 4(3):72-75.

25. Subbarao SK, Vasantha K, Raghavendra K, Sharma VP, Sharma GK: Anopheles culicifacies: siblings species composition and its relationship to malaria incidence. J Am Mosa Control Assoc 1988, 4(1):29-33.

26. Barik TK, Sahu B, Swain V: A review on Anopheles culicifacies: from bionomics to control with special reference to Indian subcontinent. Acta Trop 2009, 109(2):87-97.

27. Maheswary NP, Habib MA, Elias M: Incrimination of Anopheles aconitus Donitz as a vector of epidemic malaria in Bangladesh. Southeast Asian J Trop Med Public Health 1992, 23(4):798-801.

28. Maheswary NP, Khan Z, Molla FR, Haq MI: Incrimination of Anopheles annularis van der Wulp-1854 as an epidemic malaria vector in Bangladesh. Southeast Asian J Trop Med Public Health 1993, 24(4):776-778.

29. Reid JA: Two forms of Anopheles philippinensis in Malaya. J Med Entomol 1967, 4(2):175-179

30. Prakash A, Bhattacharyya DR, Mohapatra PK, Mahanta J: Potential of Anopheles philippinensis-nivipes complex mosquitoes as malaria vector in north-east India. J Environ Biol 2005, 26(4):719-723.

31. Bhattacharyya DR, Prakash A, Sarma NP, Mohapatra PK, Singh S, Sarma DK, Kalita MC, Mahanta J: Molecular evidence for the involvement of Anopheles nivipes (Diptera: Culicidae) in the transmission of Plasmodium falciparum in north-eastern India. Ann Trop Med Parasitol 2010, 104(4):331-336.

32. Amerasinghe PH, Amerasinghe FP, Konradsen F, Fonseka KT, Wirtz RA: Malaria vectors in a traditional dry zone village in Sri Lanka. Am J Trop Med Hyg 1999, 60(3):421-429.

33. Beier JC, Perkins PV, Koros JK, Onyango FK, Gargan TP, Wirtz RA, Koech DK, Roberts CR: Malaria sporozoite detection by dissection and ELISA to assess infectivity of afrotropical Anopheles (Diptera: Culicidae). J Med Entomol 1990, 27(3):377-384.

34. Fontenille D, Meunier JY, Nkondjio CA, Tchuinkam T: Use of circumsporozoite protein enzyme-linked immunosorbent assay compared with microscopic examination of salivary glands for calculation of malaria infectivity rates in mosquitoes (Diptera: Culicidae) from Cameroon. J Med Entomol 2001, 38(3):451-454.

35. Somboon P, Morakote N, Koottathep S, Trisanarom U: Detection of sporozoites of Plasmodium vivax and Plasmodium falciparum in mosquitoes by ELISA: false positivity associated with bovine and swine blood. Trans R Soc Trop Med Hyg 1993, 87(3):322-324.

36. Tassanakajon A, Boonsaeng V, Wilairat P, Panyim S: Polymerase chain reaction detection of Plasmodium falciparum in mosquitoes. Trans $R$ Soc Trop Med Hyg 1993, 87(3):273-275.

37. Durnez L, Van Bortel W, Denis L, Roelants P, Veracx A, Trung HD, Sochantha T, Coosemans M: False positive circumsporozoite protein ELISA: a challenge for the estimation of the entomological inoculation rate of malaria and for vector incrimination. Malaria J 2011, 10:195

38. MIS Report. http://www.nmcp.info/index.php? option=com_content\&view=article\&id=12\&ltemid $=17$.

\section{doi:10.1186/1756-3305-5-150}

Cite this article as: Alam et al: Diversity of anopheline species and their Plasmodium infection status in rural Bandarban, Bangladesh. Parasites \& Vectors 2012 5:150

\section{Submit your next manuscript to BioMed Central and take full advantage of:}

- Convenient online submission

- Thorough peer review

- No space constraints or color figure charges

- Immediate publication on acceptance

- Inclusion in PubMed, CAS, Scopus and Google Scholar

- Research which is freely available for redistribution

Submit your manuscript at www.biomedcentral.com/submit
C BioMed Central 